\title{
BCBioconjugate Chemistry
}

Subscriber access provided by BIBLIOTECA UNIVERSIDAD COMPLUTENSE DE MADRID

\section{Article}

\section{Building Block Based Construction of Membrane-Organelle Double Targeted Nanosystem for Two-Drug Delivery}

\author{
Rafael R Castillo, Daniel Lozano, and María Vallet-Regí
}

Bioconjugate Chem., Just Accepted Manuscript • DOI: 10.1021/acs.bioconjchem.8b00603 • Publication Date (Web): 01 Oct 2018

Downloaded from http://pubs.acs.org on October 2, 2018

\section{Just Accepted}

"Just Accepted" manuscripts have been peer-reviewed and accepted for publication. They are posted online prior to technical editing, formatting for publication and author proofing. The American Chemical Society provides "Just Accepted" as a service to the research community to expedite the dissemination of scientific material as soon as possible after acceptance. "Just Accepted" manuscripts appear in full in PDF format accompanied by an HTML abstract. "Just Accepted" manuscripts have been fully peer reviewed, but should not be considered the official version of record. They are citable by the Digital Object Identifier (DOI®). "Just Accepted" is an optional service offered to authors. Therefore, the "Just Accepted" Web site may not include all articles that will be published in the journal. After a manuscript is technically edited and formatted, it will be removed from the "Just Accepted" Web site and published as an ASAP article. Note that technical editing may introduce minor changes to the manuscript text and/or graphics which could affect content, and all legal disclaimers and ethical guidelines that apply to the journal pertain. ACS cannot be held responsible for errors or consequences arising from the use of information contained in these "Just Accepted" manuscripts. 


\section{INTRODUCTION}

Cancer therapy with nanotechnology has been enormously based on the Enhanced Retention and Permeation Effect (EPR) discovered in the mid-8os by Matsumura and Maeda. This effect is a spontaneous preferential accumulation of macromolecules and nanometric materials within solid tumors. This occurs due to a combination of two tumor-specific effects: first an excessive and aberrant vascularization that allows the particles to escape from the blood vessel and second, an absence of lymphatic drainage that prevents their elimination. This phenomenon has been widely employed to efficiently deliver nanoencapsulated chemotherapeutics to tumor areas while minimizing the off target drug uptake. ${ }^{2}$ These results have been successfully exported to clinical practice, reducing both side effects and dosage of chemotherapy. However, the development of new nanomedicines require from increased efficiencies able to outrange the lone effect of EPR. ${ }^{3}$ Currently, one promising trend is to turn nanomedicines from passively accumulated carriers to engineered devices able to actively address particular cell populations onto which exert their apoptotic effect. ${ }^{4}$ Regarding the targeting approach, many examples have been reported using antibodies and aptamers as targeting moieties; ${ }^{5-8}$ unfortunately, the high associated costs and low chemical stability of antibodies and the limited number of available aptamers penalize their use as all-purpose targeting tags. These drawbacks have increased the interest for more robust elements based on small molecules and peptides, which despite having lower specificity, are highly compatible with most of production processes: chemical modification, thermal treatments, sterilization processes, etc. Another important approach for the development of anticancer agents is the maximization of their apoptotic effect, ${ }^{9}$ which could be achieved by the simultaneous application of independent proapoptotic therapies. ${ }^{10-14}$ For instance, photothermal and photodynamic therapies, ${ }^{14,}{ }^{15}$ siRNAs gene expression disruption, ${ }^{16}$ immunotherapy ${ }^{17}$ and hyperthermia ${ }^{18,19}$ in combination with classic chemotherapeutic compounds. However, the combination of anticancer drugs species remains as one of the approaches with more potential and yet less exploited; issue which could be achieved with properly engineered nanocarriers. $^{20-23}$

Focusing on the construction of multi-targeted delivery systems, tissue-cell or cell-cell sequential targeting would be more interesting for homing carriers towards tumors with poor or without EPR effect, while the cell-organelle targeting may be of preference for the treatment of multidrug resistant tumors or those which show a strong EPR 
effect. In short, the choice of the different targeting units must be adapted to the chosen disease. Additionally in order to achieve improved antiproliferative effects and due to the growing problem of drug resistance, ${ }^{24}$ it has become of extraordinary importance the development of drug delivery systems able to disrupt cell replication at different points. ${ }^{13,25-27}$ To try to outrange the limitations of current nanomedicine, many different approaches based on different materials came to light; ${ }^{23}$ but among them, mesoporous silica nanoparticles (MSNs) ${ }^{28-33}$ are of special interest because of their unique porous and compartmentalized structure that permits to load drugs within the pores without affecting the surface. Interesting examples dealing with combination therapy employing MSNs could be found on a recent review. ${ }^{14}$ Therein, and as far as we know, there are no examples with double-targeted devices able to deliver two (or more) drugs; which is relevant to establish the potential of these kind of multifunctional systems.

The usual approaches for the development of particles with dual targeting properties are fundamentally limited to systems obtained upon random surface functionalization. ${ }^{14,34,} 35$ Other promising strategy employs Janus type MSNs functionalized with different molecules in each hemisphere. ${ }^{36}$ Nevertheless, both strategies lead to uncertainty in both composition and ligand ratio, making the results also subject to certain randomness. Therefore, to obtain optimal targeting results, it is of capital importance to reduce this uncertainty. We believe that this problem could be solved with the creation multifunctional building blocks that after a proper assembly onto particles, would provide those with all the functionalities.

\section{RESULTS AND DISCUSSION}

Based on the absence of knowledge about the potential behavior of multifunctional targeted nanosystems for multiple cytotoxic delivery, we decided to tackle the topic using a building block methodology that allowed to incorporate those multiple functionalities. To address this proof of concept we focused on the sequential cellorganelle targeting approach, which could be validated with simple $2 \mathrm{D}$ cell cultures.

According to our initial premise of employing small molecules, a preliminary bibliographic study restricted the candidates to: folic acid, biotin, RGD, TAT and KALA peptides for cellular targeting ${ }^{5,6}$ and triphenyl phosphonium cations (TPP), L-carnitine, dexamethasone, colchicine and some specific peptides for organelle targeting. ${ }^{37,38}$ Among those, because of synthetic simplicity and previous experimental experience, we decided to carry on our studies with folic acid, biotin and TPP as reference targeting units.

In order to implement a sequential dual cell-organelle targeting strategy, ${ }^{39}$ the most logic approach is that which places the membrane recognition fragment in the outermost part of the particle and the organelle binding element in a more internal position. This configuration is expected to produce maximal cellular uptake given the increased exposure of the membrane ligand. Moreover, is also expected that the cationic TPP fragment may facilitate interaction between ligands and negatively charged membranes.

With this layered assembly, the incorporation of additional functionalities could be achieved by employing suitable building blocks. For example, the incorporation of a cytotoxic compound could be performed by simply adding an additional, more internal, building block containing the drug. An important advantage of this strategy is that only the surface of MSNs is comprised, which leave the mesopores unoccupied, allowing the loading of a second cytotoxic drug (Figure 1).

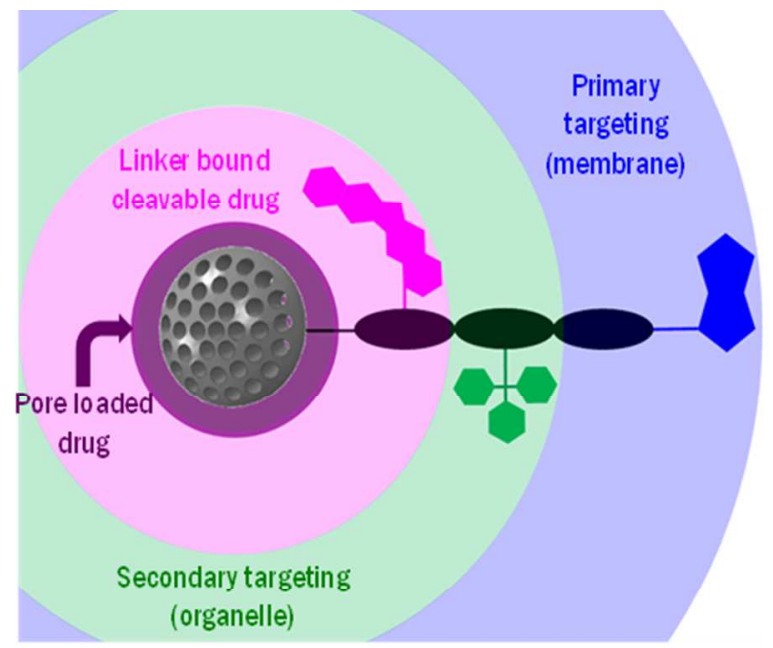

Figure 1. The multilayer strategy employed for the development of dual targeted two-drug nanocarriers based on mesoporous silica nanoparticles. 
The starting amino-capped fluorescent MSNs $\left(F^{*} M S N\right.$ $\mathrm{NH}_{2}$ ) were obtained following a two-step methodology. First, there were prepared the corresponding fluorescentlabelled MSNs following a modified Stöber methodology and then were reacted with 3-aminopropyl triethoxysilane to incorporate the corresponding amino functionality. Finally, the surfactant template was removed throughout acidic extraction steps with ethanolic $\mathrm{NH}_{4} \mathrm{NO}_{3}$ to provide the desired MSNs. The physicochemical characterization of the prepared material was in agreement with the values obtained for porous MCM-41 type materials. The porosity of the material was determined by $\mathrm{N}_{2}$ adsorption isotherm employing the Brunnauer-Emmet-Teller (BET) approach to determine surface area $\left(745.2 \mathrm{~m}^{2} \mathrm{~g}^{-1}\right)$ which followed a type IV isotherm. The Barrett-Joyner-Halenda (BJH) calculation also provided pore diameter distributions optimal for small-molecule hosting $(2.4 \mathrm{~nm})$. The porosity of the material was also corroborated in the step prior to loading, finding that the $F^{*} M S N$-Lys-(SSLinker-CPT)Lys(TPP)GABA-Biotin hybrid was still suitable for small molecule hosting, $\left(2.53 \mathrm{~nm}, 412.9 \mathrm{~m}^{2} \mathrm{~g}^{-1}\right.$, Figure $\left.\mathrm{S} 1\right)$ despite the expected pore volume reduction induced by the heavy surface functionalization (Figure $\mathrm{S}_{1}$ ). The IR analysis of all prepared Mesoporous Organosilica Hybrids (MOSHs) showed the typical Mesoporous Silica adsorption bands and the expected modifications for each new incorporated moiety (Figure S8). The small-angle X-ray scattering of $F^{*} \mathrm{MSN}-\mathrm{NH}_{2}$ showed the typical long-range ordering diffraction pattern for MCM-41 mesoporous silica with the 100,110 and 200 peaks as the most representative. Again, this pattern was maintained throughout the whole synthetic process, thus demonstrating that porosity was not affected (Figure S2). The particle size, morphology and ordered MCM-41-type pattern of the prepared $F^{*} M S N$ $\mathrm{NH}_{2}$ could be also observed on the TEM images included in the supporting information (Figures S6, S7).

The dynamic-light scattering (DLS) analysis of the different hybrids showed hydrodynamic diameter values starting from $200 \mathrm{~nm}$ of the $F^{*} \mathrm{MSN}-\mathrm{NH}_{2}$ with logic increases along with the functionalization with the different targeting fragments (Figure 2). Despite there are found slight variations for average measurements for Folic and TPP substituted particles, the raw data show comparable sizes according to the magnitude of the incorporated modification (Figure S3). Regarding the z-potential, there could be observed that all hybrids show positive values, according to the nature of the targeting moieties employed. For single targeted hybrids, the highest values of were obtained for TPP $(+32.6 \mathrm{mV})$ and Folic acid $(+32.5$ $\mathrm{mV}$ ) substitutions, while biotin modification provided a lower value $(+18 \mathrm{mV})$. In the case of double targeted hybrids, the potential values decreased as the separation between TPP and biotin augmented. This is a logic effect as the TPP, placed on more internal position is more shielded as the spacer magnitude is increased (Figure 2). All these hybrids have outstanding dispersibility consequence of their functionalization with highly hydrophilic fragments.

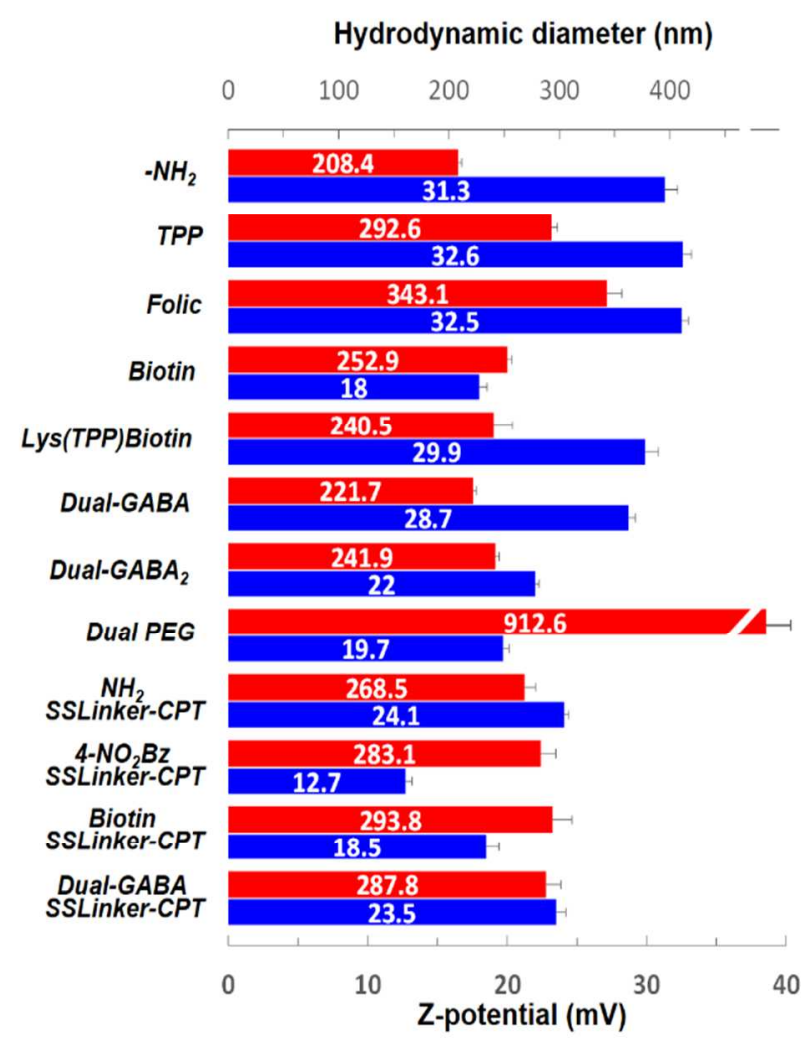

Figure 2. Hydrodynamic diameters (red) and $\mathrm{z}$ potential values (blue) obtained by Dynamic Light Scattering (DLS) for all reported hybrids. Data showed correspond to a mean of 5 measures and were recorded in distilled water. Please check Figures $\mathrm{S}_{3}$ and $\mathrm{S}_{4}$ in the supporting information for raw data of these measurements. Dual refers to TPP-Biotin double targeted ligands with the indicated connectivity. Values for DOX-loaded particles could be found on figure $\mathrm{S}_{5}$.

The incorporation of the cleavable CPT-containing ( $\mathrm{HO}$ SSLinker-CPT) fragment, although in a more internal layer, affected both hydrodynamic size and surface potential. As could be seen on figure 2 , size values were increased in about $40 \mathrm{~nm}$ for CPT-modified particles for comparable cases (biotin and Lys(TPP)GABA-Biotin). Something similar occurred with the z-potential value, probably due to the hydrophobic contribution of SSLinker-CPT, which in the case of Lys(TTP)GABA-Biotin hybrid led to a potential decrease of about $5 \mathrm{mV}$. In any case, the similar values obtained between CPT-containing and non-containing particles seem to indicate that the internal Lys(SSLinker-CPT) modification is placed far from the outer shell and ensures an appropriate exposition of the targeting moieties (Figure 2).

The thermogravimetric analysis (TGA) of the different hybrids clearly showed an organic matter increase form the $12 \%$ of $F^{*} \mathrm{MSN}-\mathrm{NH}_{2}$ to about $20 \%$ for mono-targeted hybrids (Figure S9, up). This pattern is however not maintained in the case of bifunctional ligands. In these cases, was observed that Lys(TPP)PEG-Biotin $(\approx 26 \%)$ and nonspaced bifunctional Lys $($ TPP $)$ Biotin $(\approx 23 \%)$ modifications leaded to greater mass losses than the two GABA- 
containing hybrids $(\approx 17$ and $21 \%)$. Those variations could be understood considering the growing steric hindrance of ligands, which eventually may block some anchoring positions. Regarding the PEG-spaced bis-targeted materi-

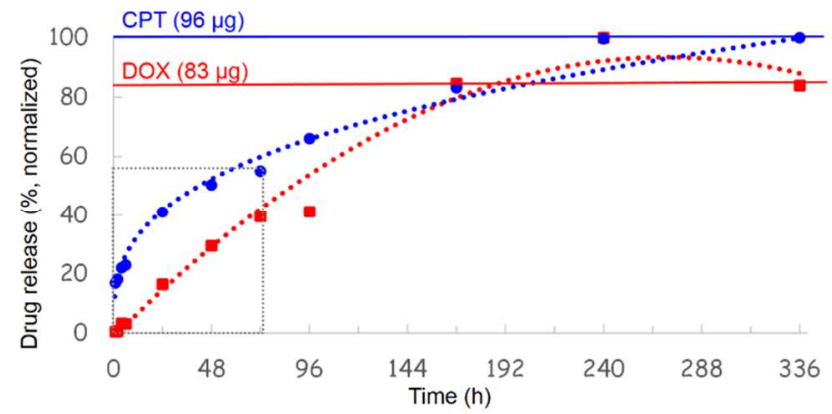

Figure 3. Simultaneous drug release obtained for DOX@F*MSN-Lys(SSLinker-CPT)-Lys(TPP)GABA-Biotin obtained at $37^{\circ} \mathrm{C}$ during 14 days. The $\mu$ g quantities noted on the release graph express the interpolated release values adjusted for the actual amount of hybrid employed. The calculated $96 \mu \mathrm{g}$ estimated for CPT, correspond to a functionalization yield of about $9 \%$ (referenced to the maximum number of available $\mathrm{NH}_{2}$ groups, supporting information).

al, there should be accounted that the employed 27 mer PEG has an enormous organic matter contribution and that was synthetized following a different strategy, which makes its comparison poorly relevant (Figure S9, down). The analysis of the drug containing $F^{*} M S N$-Lys-(SSLinkerCPT)-Lys(TPP)GABA-Biotin hybrid behaved as expected, increasing the organic matter by $8 \%$. The second drug loading process, the incorporation of DOX into mesopores, increased again the decomposable matter in an additional 6\% (Figure Sio).

As will be discussed below, the two-drug system designed is capable of producing cell death with high efficiency. However, given that the release of both drugs does not occur simultaneously, it is also important to know the release patterns of both species. To determine the fate of both drugs there were carried out comparative experiments in a simulated intracellular environments with the DOX@F*MSN-Lys(SSLinker-CPT)Lys(TPP)GABABiotin hybrid. According to the described procedure the release experiment was carried out in $10 \mathrm{mM}$ DTT containing PBS $(\mathrm{pH}=7.4)$ to mimic the presence of intracellular Glutathione. The results showed different release profiles for both drugs. DOX, at the pores, showed a sustained, almost linear release $(17,30$ and $40 \%$ at 24,48 and $72 \mathrm{~h}$ ); while CPT, grafted on the surface throughout a cleavable linker, suffered from a potential-like, faster cleavage (40, 50 and $55 \%$ for the same times). Regardless of the release kinetics followed by each drug, it is observed that complete release was reached at about $24 \mathrm{oh}$ (Figure 3).

The tested MOSHs were prepared from $F^{*} \mathrm{MSN}-\mathrm{NH}_{2}$ in a single coupling step with the corresponding mono- or bifunctional ligands according to the experimental procedure detailed below. (Figure 4) The preparation of the TPP-Biotin bifunctional ligands was accomplished employing the orthogonally protected Lysine supported onto Wang's resin, according to the procedure described on the supporting information.

The uptake ratio obtained for the three single targeted MOSHs on mouse pre-osteoblastic MC $\mathrm{MC}_{3}$-E1 cells and on Human Osteosarcoma (HOS) cells $\mathrm{s}^{40,41}$ showed that Biotin (ca. 50\%) targeted particles provided better internalization than folate (ca. 40\%) and TPP (ca. 40\%) targeted particles. However, since the TPP was the only organelle targeting molecule preselected, it will be included onto the bifunctional ligands (Figures 4, $\mathrm{S}_{11}-\mathrm{S}_{13}$ ). When the Lys(TPP)Biotin targeted MOSHs uptake was evaluated against HOS cell line, there was found a significant reduction of the internalization, indicating that both targeting elements (TPP and Biotin) interfered with each other on this particular setup.

To overcome this problem, there were prepared three different MOSHs with increased space between both targeting units. To make the system as much biocompatible
SINGLE TARGETED MOSHs

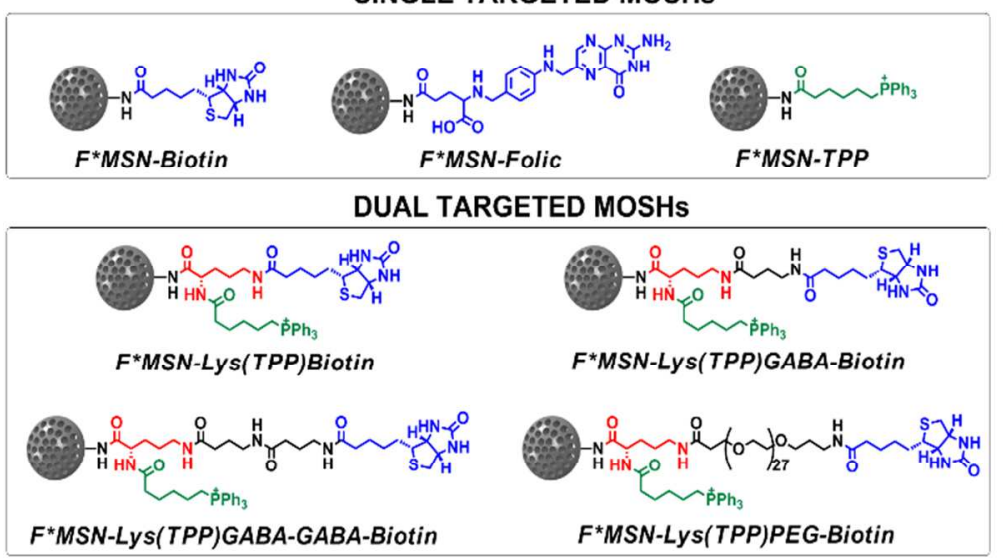

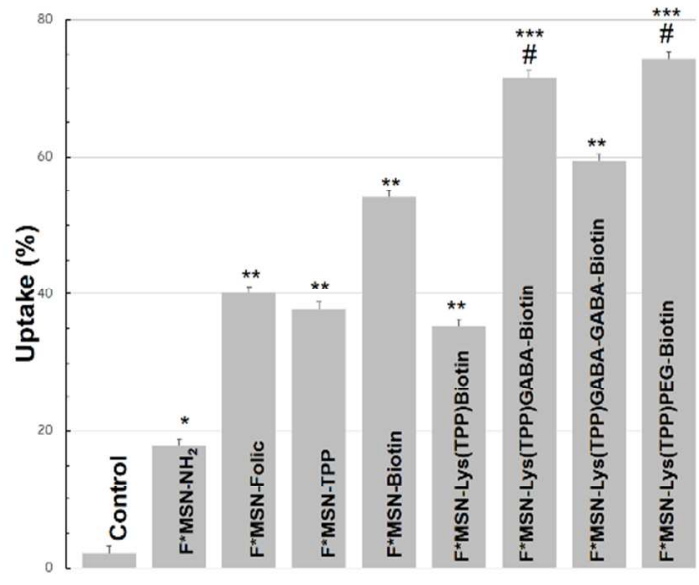

Figure 4. Prepared single and double targeted fluorescein labeled organosilica nanohybrids. Cell uptake measured by flow cytometry at $2 \mathrm{~h}$ of internalization in HOS cells. Data are mean \pm SEM of 3 independent experiments performed in triplicate ${ }^{*} \mathrm{p}<0.05$ vs HOS control; ${ }^{* *} \mathrm{p}<0.05$ vs $F^{*} M S N-N_{2} ;{ }^{* * *} \mathrm{p}<0.01 v s F^{*} M S N-N_{2} ; \# \mathrm{p}<0.01$ vs all groups. 

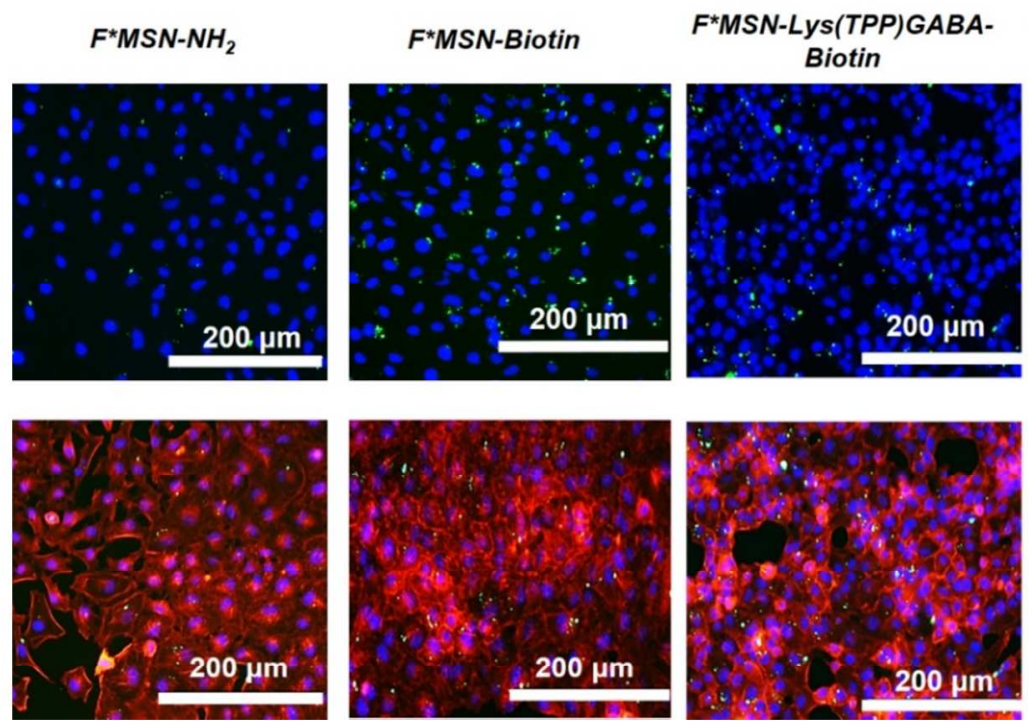

$F^{*} M S N-L y s(T P P) G A B A-$ GABA-Biotin
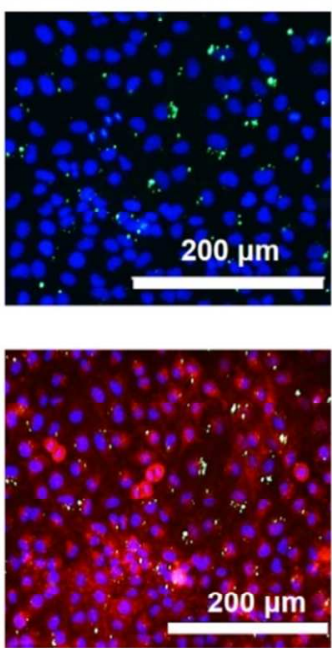

Figure 5. Representative fluorescence microscopy images of HOS cells incubated with fluorescein labeled nanoparticles at $2 \mathrm{~h}$ of internalization. Blue fluorescence (nuclei), red (actin filaments) and green fluorescence (fluorescein labeled nanoparticles).

as possible, there were chosen $\gamma$-aminobutyric acid (GABA) as short connecting unit and a bifunctional aminoacid containing PEG as long spacer $\left(\mathrm{HO}_{2} \mathrm{C}-\mathrm{PEG}_{27 \text { mer- }}{ }^{-}\right.$ $\mathrm{NH}_{2}$ ); in both cases as their protected Fmoc analogs. The preparation of the spaced dual targeted ligands could be found on the supporting information. Uptake ratio of these MOSHs showed that elongated bifunctional ligands improved overall internalization, which leaped up from less than $40 \%$ of the $F^{*} M S N-L y s(T T P)$-Biotin to more than $70 \%$ in the cases of $F^{*} M S N$-Lys(TTP)GABA-Biotin and $F^{*} M S N-L y s(T T P) P E G$-Biotin hybrids (Figures 4 and 5 ). Despite this similarity, there was found a maximal fluorescence intensity on the hybrids modified with Lys(TTP)GABA-Biotin, (Figure S13) which will be employed as reference targeting building block for the following investigations.

It is interesting to note that the incorporation of any spacer, independently from its magnitude, between both targeting units enhance internalization above the values obtained for the two single targeted particles. However, this effect does not seem to occur in the case of for $F^{*} M S N-L y s(T T P) G A B A-G A B A-B i o t i n$, which show comparable values to those obtained for Biotin mono-targeted MSNs. However this apparent diverging behavior could be explained upon analysis of thermogravimetric curves obtained for its organic matter decomposition. (Figure S9) Those clearly show lower mass loss, which indicates a lower number of ligands and therefore, lower internalization.

Once the bifunctional targeting ligand has been selected, to continue advancing in the idea of the multifunctional device, is necessary to include an additional building block at the surface to allow the inclusion of a drug. Among all antiproliferative drugs, there were chosen Camptothecin (CPT) and Doxorubicin (DOX) as two reference antitumor agents with specificity against topoisomerases I and II respectively; from which may be expected an enhanced cell death induction when combined. To facilitate the incorporation of both antitumor species, we decided to load DOX into the pores while CPT would be incorporated in a new building block at the surface. The chosen drug location obeys to the nice behavior shown by DOX in both of loading and release processes from silica's mesopores, as recurrently demonstrated on the literature.

In order to maximize the effect of the surface-bounded drug two premises must be complied: first the drug should be bounded throughout a cleavable bond to facilitate its release and second, this bond must not affect its activity. The advantages of employing CPT plus a redoxsensitive traceless connection ${ }^{42}$ are the following: a low number of functional groups which reduce the possibility of side reactions, CPT is recovered in its active form, intracellular release is the only possibility and finally, the active region of CPT is not altered. The preparation of the cystamine-based cleavable linker ${ }^{43}$ and its later coupling with CPT (HO-SSLinker-CPT) is detailed on the supporting information.

The construction of targeted hybrid systems modified with CPT was made from Lys(Fmoc)Mtt functionalized MOSHs which were submitted to Mtt deprotection and coupled with the corresponding ligand through the terminal amino moiety; then after Fmoc cleavage, the $\mathrm{HO}$ SSLinker-CPT building block was bound to the $\alpha-\mathrm{NH}_{2}$ moiety. The non-targeted hybrid ( $F^{*} M S N-L y s$ (SSLinkerCPT) $\mathrm{NH}_{2}$ ) was prepared in a three-step sequence involving Fmoc cleavage, coupling with HO-SSLinker-CPT and Mtt deprotection. Next, the CPT-containing hybrids $\left(F^{*} M S N\right.$-Lys(SSLinker-CPT)-Ligand) and their equivalent analogs without such modification, $\left(F^{*} M S N\right.$-Ligand) were loaded with DOX and subjected to viability assay (Figure 6).

As expected, it was found that systems without drugs led to higher viabilities, perhaps reduced a membrane desta- 


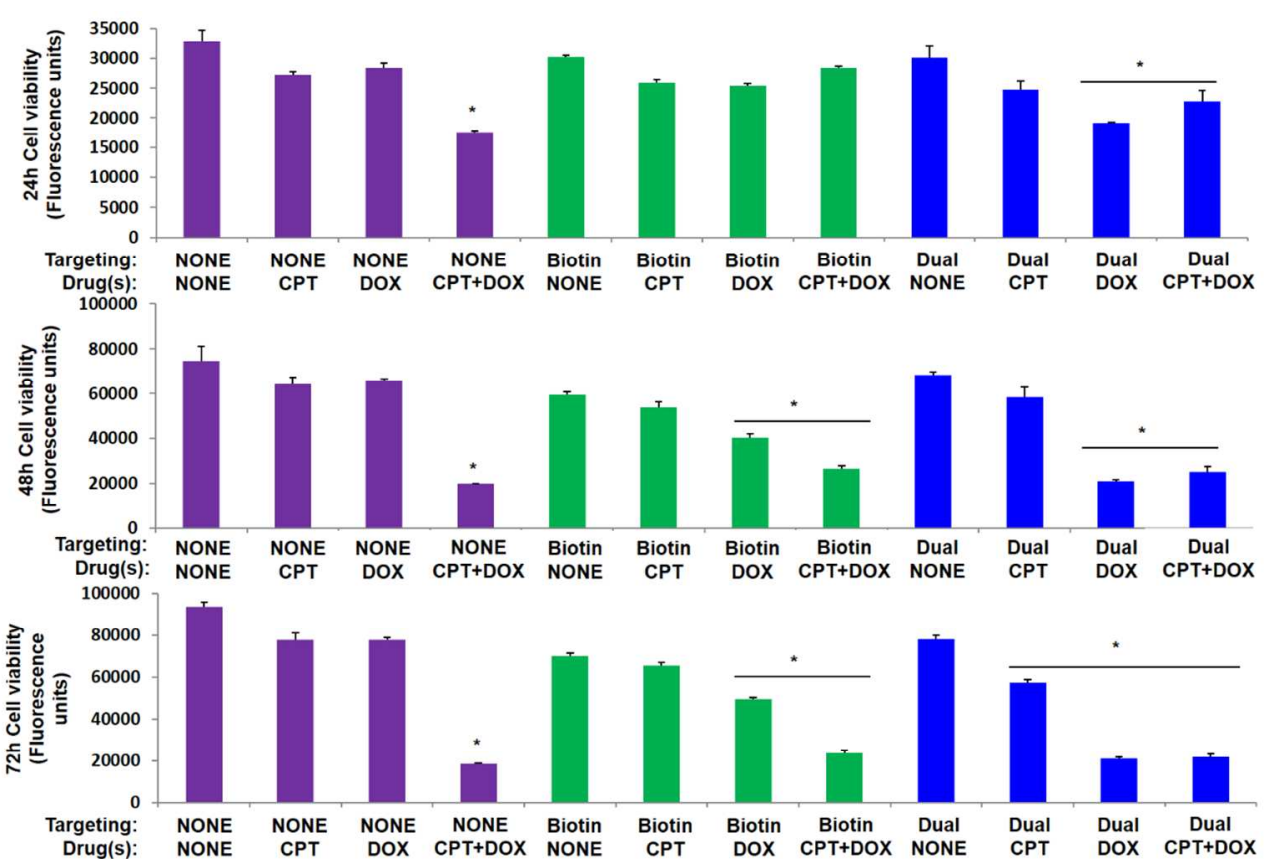

Figure 6. Cytotoxicity assay measured by Alamar Blue in HOS cells with the different nanoparticles loaded with DOX, CPT or both at $24 \mathrm{~h}(\mathrm{~A}) 48 \mathrm{~h} \mathrm{(B)}$ and $72 \mathrm{~h}(\mathrm{C})$ of cell culture. Data are mean \pm SEM of 3 independent experiments performed in triplicate. ${ }^{*} \mathrm{p}<0.05 v s$ corresponding non-targeted, Biotin or dual nanoparticles. NONE/DOX, particles refer to DOX loaded $F^{*} M S N-N H_{2}\left(D O X @ F^{*} M S N-N H_{2}\right)$, NONE/CPT refers to $F^{*} M S N-L y s(S S L i n k e r-C P T) N H_{2}$ and NONE/CPT+DOX to $D O X @ F^{*} M S N-L y s(S S L i n k e r-C P T) N H_{2}$. Notation employed for other hybrids is equivalent, with the proviso that Dual refers to Lys(TPP)GABA-Biotin targeting ligand.

bilizing effect attributable to the TPP; whilst the presence of cytotoxic agents increased the mortality. In the case of systems including a single cytotoxic species, a substantial reduction in cell death was hardly achieved, which may point out drug-resistance. Regarding the case of two-drug systems there was obtained a marked death induction, which seems to indicate a cooperative apoptotic effect. It is noteworthy that although complete drug release was achieved within 10 days, the synergic therapeutic effect came earlier. Indeed, after $48 \mathrm{~h}$, a very sharp drop in viability could be observed (Figures 6 and 7 ). This effect, which seem to be aligned with a significant release of both DOX and CPT (30 and 50\% of the total load), pointed out that in less than $24 \mathrm{~h}$, only CPT apoptotic mechanism operated. The similar viability values found for 48 and $72 \mathrm{~h}$ suggest that both optimal concentration of CPT and DOX was reached during the first two days of incubation.

It is also remarkable that two examples moved away from the expected mortality trend: the non-targeted, twodrug hybrid and the system that combines DOX and TPPBiotin Dual Ligand (Figure 6). The first result could be understood considering that the free amino groups at the surface could produce a non-specific uptake upon interaction with the membranes. To demonstrate this postulate there was also prepared a MOSHs bearing an inactive targeting, the neutral but polar 4-Nitrobenzoic acid. When this non-active targeted $F^{*} M S N$-Lys(SSLinkerCPT) $\mathrm{NO}_{2}$ hybrid was compared with the other targeted devices, there was found an enhanced cell survival. (Figure 7) This highlights an unspecific amino-mediated uptake and proves the importance of targeting to maximize tumor-delivery. Unfortunately, since this unspecific electrostatic induced uptake cannot be used to discriminate between different cell types, it is of little interest for the development of anticancer therapeutics. The second diverging result, single-drug DOX chemotherapeutic in combination with the dual ligand Lys(TPP)GABA-Biotin, is harder to explain because mitochondrial targeting may be acting as destabilizing agent and thus enhancing mortality. In any case, this result also provides invaluable information since it validates the efficiency of our dual cellular-organelle targeted nanodevice for the design of more efficient, new generation, drug delivery systems.

\section{CONCLUSIONS}


Despite the use of nanoparticles in medicine has opened new possibilities for advanced cancer treatment, the reality is that a surface functionalization is required to reach their maximum potential. On this contribution we describe a multifunctional two-drug, double vectored nanodevice based on Mesoporous Silica, which was built by layered assembly of different functional building blocks. This system, which according to our knowledge is the first of its kind, proposes a sequential cell-organelle targeting plus two-drug delivery. This dual release is based on combining spontaneous diffusion from a poreloaded drug (DOX) together with a dithiol-mediated cleavage of a surface-bound antitumor drug (CPT). Moreover it has been optimized a novel membrane-organelle dual targeting ligand, which has demonstrated to be more efficient than independent targeting fragments. This highly positive result opens an access point to the development of more double-targeted ligands employing more specific components. The in vitro results of internalization and viability against HOS cells demonstrate that this hybrid system shows its best performance at $48 \mathrm{~h}$, where the maximal apoptosis induction is obtained. In view of these results, the future work will deal with the implementation of this two-drug delivery system with high affinity targeting moieties, the implementation of a triple tissuemembrane-organelle targeting ligands and in vivo testing.

\section{EXPERIMENTAL SECTION}

Reagents and equipment: Ammonium nitrate, Cetyltrimethylammonium bromide (CTAB), Tetraethyl orthosilicate (TEOS), Biotin, Folic acid, 1Hydroxybenzotriazole (HOBt), $N, N, N^{\prime}, N^{\prime}$-Tetramethyl-O(1H-benzotriazol-1-yl)uronium hexafluorophosphate (HBTU), 4-Nitrobenzoic acid, N-(3Dimethylaminopropyl)- $N$ '-ethylcarbodiimide hydrochloride (EDC), 4-(Fmoc-amino)butyric acid (Fmoc-GABA$\mathrm{OH}), \quad \mathrm{O}$-(2-Carboxyethyl)-O'-[2-(Fmoc-amino)ethyl]heptacosaethyleneglycol (HO-PEG $\left.{ }_{27 m e r}-\mathrm{NHFmoc}\right)$, (4-Carboxybutyl)-triphenylphosphonium bromide (TPP), diisopropylethylamine (DIPEA), 4-dimethylaminopyridine
(DMAP), (S)-(+)-Camptothecin (CPT), DL-Dithiothreitol (DTT) and Sephadex G-25 were purchased from SigmaAldrich; from ABCR were obtained 3aminopropyltriethoxysilane (APTES), Fluorescein isothiocyanate (FITC) and Doxorubicin hydrochloride (DOX). The two lysine modified reagents: free and Wang's resinbounded $\quad N$ - $\alpha$-Fmoc- $N$ - $\varepsilon$-4-methyltrityl- $L$-lysine (HOLys(Fmoc)Mtt) and [Wang]-Lys(Fmoc)Mtt) were obtained from Novabiochem. Cell culture media DMEM, Fetal Bovine Serum and trypsin were purchased from Life Technologies while the Alamar Blue solution from AbD Serotec. All compounds and solvents were employed without additional purification. Prepared materials were analyzed employing the available instruments: Philips X-Pert MPD diffractometer equipped with $\mathrm{CuK}_{\alpha}$ radiation for X-ray diffraction (XRD), Perkin-Elmer Pyris Diamond TG/DTA analyzer for thermogravimetry and differential temperature analyses (TGA/DTA), Nicolet Nexus (Thermo Scientific) spectrometer equipped with a Smart Golden Gate ATR accessory for Fourier transform infrared (FTIR) spectra and a Bruker AV $250 \mathrm{MHz}$ for NMR experiments. Freeze-dying was carried out in a Telstar LyoQuest apparatus. Particles' morphology was analyzed by transmission electron microscopy (TEM) was carried using a JEOL JEM 2100 microscope equipped with a CCD camera (KeenView Camera). Pore properties were determined by $\mathrm{N}_{2}$ adsorption isotherms on a Micromeritics ASAP 2020 instrument; the surface area was obtained by applying the Brunnauer, Emmet \& Teller (BET) method to the isotherm while pore size distribution was determined by the Barrett, Joyner \& Halenda $(\mathrm{BJH})$ method from the desorption branch of the isotherm. The zeta potential and hydrodynamic diameter of nanoparticles were measured by means of a Zetasizer Nano ZS (Malvern Instruments) equipped with a $633 \mathrm{~nm}$ laser. Ultraviolet-visible spectra were recorded in a Helios Zeta or Unicam UV-50o spectrometers from Thermo Scientific employing $1 \mathrm{~cm}$ optical way, reduced volume cuvettes. Fluorescence microscopy was performed on an Evos FL Cell Imaging System equipped with tree led channels (DAPI/GFP/RFP) from AMG (Advance Microscopy Group). Fluorescence spectrometry for drug release

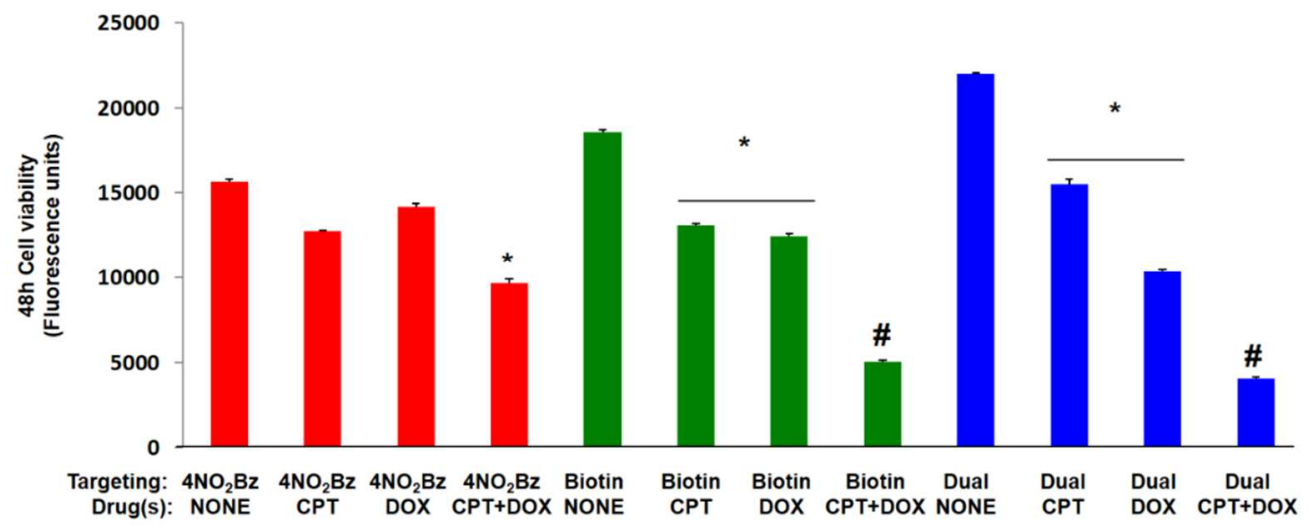

Figure 7. Cytotoxicity assay measured by Alamar Blue in HOS cells for differently targeted $\mathrm{MOSHs}\left(4-\mathrm{NO}_{2} \mathrm{Bz}\right.$, Biotin and Lys(TPP)GABA-Biotin) with DOX, CPT or both at $48 \mathrm{~h}$ of cell culture. Data are mean \pm SEM of 3 independent experiments performed in triplicate. ${ }^{*} \mathrm{p}<0.05$ vs corresponding $4-\mathrm{NO}_{2} \mathrm{Bz},(4$-Nitrobenzoyl) Biotin or dual targeted hybrids; \#p < o.ool vs corresponding 4-NO $\mathrm{NO}_{2} \mathrm{Bz}$, Biotin or dual targeted hybrids. Dual refers to Lys(TPP)GABA-Biotin targeting ligand. 
studies was performed in a synergy 4 microplate reader from BioTek. Flow cytometry data were obtained from a FACScan machine (Becton, Dickinson and Company, USA).

Synthesis of amino-capped fluorescent Mesoporous Silica Nanoparticles: $F^{*} \mathrm{MSN}-\mathrm{NH}_{2}$ nanoparticles were synthesized employing a modified Stöber methodology in which a mixture of TEOS $(5 \mathrm{~mL})$ and fluorescein labeled APTES ( 1 mg FITC plus $2.2 \mu \mathrm{L}$ APTES reacted in $20 \mu \mathrm{L}$ ethanol for $2 \mathrm{~h}$ ) were dropwise added $\left(0.25 \mathrm{~mL} \mathrm{~min}^{-1}\right)$ over a heated $\left(80^{\circ} \mathrm{C}\right)$ solution of CTAB $(1 \mathrm{~g})$ and $\mathrm{NaOH}(2 \mathrm{M}$, $3.5 \mathrm{~mL}) 48 \mathrm{o} \mathrm{mL}$ in bidistilled water under stirring. Once the addition was concluded, the mixture was stirred for 2 additional hours to finish the silica condensation. Then, the mixture was submitted to usual centrifugation step (100oo rpm, $10 \mathrm{~min}$ ) and repeated washings with water $(2 \times 200 \mathrm{~mL})$ and ethanol $(2 \times 100 \mathrm{~mL})$ to remove the excess of reagents. The obtained fluorescent MSNs were then dispersed in EtOH (500 mL) and reacted with APTES (200 $\mu \mathrm{L})$ at $45^{\circ} \mathrm{C}$ for $24 \mathrm{~h}$ to functionalize the outer silica surface. Once the particles were capped with the amino moieties, the surfactant contained within the pores was removed by ionic exchange throughout 2 hour reflux cycles with extracting solution $\left(2 \times 250 \mathrm{~mL}\right.$ of a $10 \mathrm{~g} \mathrm{~L}^{-1}$ of $\mathrm{NH}_{4} \mathrm{NO}_{3}$ in 95:5 ethanol-water) followed by centrifugation. Finally, the obtained particles were washed with EtOH (3x10o mL), dried, dispersed in DMF (20 mg $\mathrm{mL}^{-1}$ ) and stored refrigerated.

Solid-phase synthesis of bifunctional ligands: The synthesis of TPP and Biotin containing ligands was accomplished throughout the solid phase methodology described on the supporting information. Briefly, the synthetic protocol involved Fmoc deprotection of the commercial [Wang]-Lys(Fmoc)Mtt resin, followed by coupling with TPP. Then, after cleavage of the Mtt protecting group were coupled the lysine with either a Fmocprotected spacer or with the terminal biotin. In the case coupling with spacer, an additional Fmoc deprotection and coupling step was accomplished. Finally the bifunctional ligand was cleaved from the resin employing a $\mathrm{TFA} / \mathrm{H}_{2} \mathrm{O} / \mathrm{Et}_{3} \mathrm{SiH}(95 / 2.5 / 2.5)$ mixture. Ligand purification was done by Sephadex G-25 size exclusion chromatography with $\mathrm{H}_{2} \mathrm{O}$ as eluent and isolated by lyophilization.

Synthesis of targeted Mesoporous OrganoSilica Hybrids (MOSHs): The syntheses of single and double targeted MOSHs was accomplished in a single step form the prepared $F^{*} \mathrm{MSN}-\mathrm{NH}_{2}$ and the appropriate mono/bifunctional ligand following one of the described coupling protocols described on the supporting information. For the PEG spaced dual targeted hybrids there was followed a stepwise procedure also detailed on the supporting information. Once the targeted MOSHs were obtained, they were isolated by centrifugation, washed with DMF (2×20 mL) and ethanol $(2 \times 20 \mathrm{~mL})$ and stored refrigerated at work concentration $\left(20 \mathrm{mg} \mathrm{mL}^{-1}\right)$ in $\mathrm{EtOH}$.

Synthesis of Camptothecin-modified targeted MOSHs: The synthesis of CPT modified hybrids started from the lysine modified particles ( $\approx 100 \mathrm{mg}$ scale) which were submitted to a two-step deprotection-coupling sequence. Briefly the followed steps include Mtt deprotection, coupling with the corresponding ligand, (4nitrobenzoic acid, biotin or HO-Lys(TPP)GABA-Biotin), Fmoc cleavage and finally, coupling with the Camptothecin dithiol linker. Protocols are detailed on the supporting information.

Loading protocol for Doxorubicin: For the optimal diffusion of the drug in between the mesopores, $10 \mathrm{mg}$ of the corresponding MOSHs were set under vacuum at $80^{\circ} \mathrm{C}$ for $2 \mathrm{~h}$ to remove remainder solvent and volatile compounds. To the vacuumed MOSHs were added $2 \mathrm{~mL}$ of a saturated solution of DOX in $\mathrm{MeOH}$, this mixture was kept for $24 \mathrm{~h}$ at RT to allow full diffusion throughout the mesopores. After completion, the loaded particles were centrifuged, washed with $\mathrm{MeOH}(2 \times 2 \mathrm{~mL})$ and $\mathrm{H}_{2} \mathrm{O}(5 \times 2$ $\mathrm{mL})$ to remove DOX excess. The particles were stored with refrigeration as centrifuged and used immediately.

Drug release studies: Drug release studies were carried out with a pur-A-lizer ${ }^{\mathrm{TM}}$ mini 6000 dialysis kit filled with a suspension of DOX-loaded $F^{*} M S N-L y s(S S L i n k e r-C P T)$ Lys(TPP)GABA-Biotin in bidistilled water $\left(3 \mathrm{mg} \mathrm{mL}^{-1}, 1\right.$ $\mathrm{mL}$ ). The filled kit was inserted into a reaction vessel containing $10 \mathrm{~mL}$ of $0.1 \mathrm{M}$ solution of DTT in PBS. From the supernatant were taken aliquots $(200 \mu \mathrm{L})$ at indicated times for fluorescence measurements of released CPT $\left(\lambda_{\mathrm{ex}}=370 \mathrm{~nm}, \lambda_{\mathrm{em}}=434 \mathrm{~nm}\right)$ and DOX $\left(\lambda_{\mathrm{ex}}=470 \mathrm{~nm}\right.$, $\left.\lambda_{\text {em }}=595 \mathrm{~nm}\right){ }_{.}^{44,45}$

Cell cultures: Cell culture tests were performed using $\mathrm{MC}_{3} \mathrm{~T}_{3}$-E1 (mouse pre-osteoblastic cells) and HOS cells derived from a human osteosarcoma (CRL-1543; ATCC, Mannassas, VA). The different types of nanoparticles (100 $\mu \mathrm{gL}^{-1}$ ) were placed into each well of 6- or 12-well plates (Corning, CULTEK, Madrid, Spain) after cell seeding. Cells were then plated at a density of $1.5 \times 10^{4}$ cells $\mathrm{cm}^{-2}$ in Dulbecco's modified Eagle's medium (DMEM, Sigma Chemical Company), respectively, containing $10 \%$ of heatinactivated fetal bovine serum (FBS, Thermo Fisher Scientific) and $1 \%$ penicillin-streptomycin (Thermo Fisher Scientific) at $37^{\circ} \mathrm{C}$ in a humidified atmosphere of $5 \% \mathrm{CO}_{2}$, and incubated for different times. Some wells contained no nanoparticles as controls.

Cell viability studies: HOS proliferation was determined by addition of Alamar Blue solution at $10 \%(\mathrm{v} / \mathrm{v})$ to the cell culture at 24 and $48 \mathrm{~h}$ of grown, after $2 \mathrm{~h}$ of nanoparticles internalization, following manufacturer's instructions. Fluorescence intensity was measured using excitation emission wavelengths of 570 and $600 \mathrm{~nm}$ respectively, in a Unicam UV-50o UV-visible spectrophotometer.

Cell uptake studies: $\mathrm{MC}_{3} \mathrm{~T}_{3}-\mathrm{E}_{1}$ and HOS cells were cultured in each well of a 6-well plate. After 24h, the cells were incubated at different times in the absence or presence of the tested nanoparticles $\left(100 \mu \mathrm{g} \mathrm{mL}^{-1}\right)$. After $2 \mathrm{~h}$, cells were washed twice with PBS and incubated at $37^{\circ} \mathrm{C}$ with trypsin-EDTA solution (Sigma-Aldrich) for cell detachment. The reaction was stopped with culture medium after $5 \mathrm{~min}$ and cells were centrifuged at $1000 \mathrm{rpm}$ for 10 min and suspended in fresh medium. Then, the surface 
fluorescence of the cells was quenched with trypan blue (o.4\%) to confirm the presence of an intracellular, and therefore internalized, fluorescent signal. Flow cytometry measurements were performed at an excitation wavelength of $488 \mathrm{~nm}$, green fluorescence was measured at 530 $\mathrm{nm}$ (FL1). The trigger was set for the green fluorescence channel (FL1). The conditions for the data acquisition and analysis were established using negative and positive controls with the CellQuest Program of Becton-Dickinson and these conditions were maintained during all the experiments. Each experiment was carried out three times and single representative experiments are displayed. For statistical significance, at least $10^{4}$ cells were analyzed in each sample in a FACScan machine (Becton, Dickinson and Company, USA) and the mean of the fluorescence emitted by these single cells was used.

Fluorescence microscopy: HOS cells were incubated with the nanoparticles $\left(100 \mu \mathrm{g} \mathrm{mL}^{-1}\right)$ for $2 \mathrm{~h}$. Each well was washed with cold PBS for three more times to get rid of the nanoparticles not internalized into the cells, and then fixed with $75 \%$ ethanol (kept at $-20{ }^{\circ} \mathrm{C}$ ) for $10 \mathrm{~min}$. After the ethanol was suck and washed three times with cold PBS, actin filaments were stained in red with Alexa Fluor ${ }^{\circledast}$ 555 Phalloidin (Thermo Fisher Scientific) for 15 min at 1:30 in BSA. The nuclei of HOS cells were stained with 4,6Diamidine-2'-phenylindole dihydrochloride (DAPI, $\geq 98 \%$, Sigma-Aldrich) for 5 min, respectively, and then washed three times with cold PBS. Fluorescence microscopy of nanoparticles internalized by HOS cells were performed with an Evos FL microscope employing the following wavelengths $\left(\lambda_{\mathrm{ex}}(\mathrm{nm}) ; \lambda_{\mathrm{em}}(\mathrm{nm})\right)$ : DAPI $(357-44 ; 447-60)$, GFP (470-22; 525-50), RFP (531-40; 593-40). Red channel was used to label the cytoplasm, green for nanoparticles and blue for cell nucleus.

Statistical analysis: Results are expressed as mean \pm SEM. Statistical evaluation was carried out with nonparametric Kruskal-Wallis test and post hoc Dunn's test, when appropriate. A value of $\mathrm{p}<0.05$ was considered significant.

\section{ASSOCIATED CONTENT}

Supporting Information. Detailed synthetic procedures and characterization data for building blocks and particles plus additional in vitro experiments are included. This material is available free of charge via the Internet at http://pubs.acs.org/

\section{AUTHOR INFORMATION}

\section{Corresponding Authors}

rafcasoı@ucm.es (RRC), vallet@ucm.es (MVR)

\section{ORCID Numbers}

oooo-0oo3-1957-3098 (RRC), oooo-0oo1-5902-9201 (DL), oooo-ooo2-6104-4889 (MVR)

\section{Notes}

The authors declare no competing financial interest.
The authors want to acknowledge financial support from European Research Council ERC-2015-AdG-69416o] and Ministerio de Economía y Competitividad MAT2015-64831-R]

\section{REFERENCES}

(1) Matsumura, Y., Maeda, H. (1986) A New Concept for Macromolecular Therapeutics in Cancer Chemotherapy: Mechanism of Tumoritropic Accumulation of Proteins and the Antitumor Agent Smancs. Cancer Res. 46, 6387-6392.

(2) Brigger, I., Dubernet, C., Couvreur, P. (2012) Nanoparticles in cancer therapy and diagnosis. Adv. Drug Deliv. Rev. 64, 24-36.

(3) Bertrand, N., Wu, J., Xu, X., Kamaly, N., Farokhzad, O. C. (2014) Cancer nanotechnology: the impact of passive and active targeting in the era of modern cancer biology. Adv. Drug Deliv. Rev. 66, 2-25.

(4) Pérez-Herrero, E., Fernández-Medarde, A. (2015) Advanced targeted therapies in cancer: Drug nanocarriers, the future of chemotherapy. Eur. J. Pharm. Biopharm. 93, 52-79.

(5) Ruoslahti, E. (2012) Peptides as Targeting Elements and Tissue Penetration Devices for Nanoparticles. Adv. Mater. 24, 37473756.

(6) Gao, H., Zhang, Q., Yang, Y., Jiang, X., He, Q. (2015) Tumor homing cell penetrating peptide decorated nanoparticles used for enhancing tumor targeting delivery and therapy. Int. J. Pharm. 478, 240-250.

(7) Goodall, S., Jones, M. L., Mahler, S. (2015) Monoclonal antibody-targeted polymeric nanoparticles for cancer therapy future prospects. J. Chem. Technol. Biotechnol. 90, 1169-1176.

(8) Bazak, R., Houri, M., El Achy, S., Kamel, S., Refaat, T. (2015) Cancer active targeting by nanoparticles: a comprehensive review of literature. J. Cancer Res. Clin. Oncol. 141, 769-774.

(9) Jhaveri, A., Deshpande, P., Torchilin, V. (2014) Stimulisensitive nanopreparations for combination cancer therapy. $J$. Control. Release 190, 352-370.

(10) Xu, X., Ho, W., Zhang, X., Bertrand, N., Farokhzad, O. (2015) Cancer nanomedicine: from targeted delivery to combination therapy. Trends Mol. Med. 21, 223-232.

(11) Fan, W., Yung, B., Huang, P., Chen, X. (2017) Nanotechnology for Multimodal Synergistic Cancer Therapy. Chem. Rev. 117, 13566-13638.

(12) Gadde, S. (2015) Multi-drug delivery nanocarriers for combination therapy. Med. Chem. Commun. 6, 1916-1929.

(13) He, C., Tang, Z., Tian, H., Chen, X. (2016) Co-delivery of chemotherapeutics and proteins for synergistic therapy. $A d v$. Drug Deliv. Rev. 98, 64-76.

(14) Castillo, R. R., Colilla, M., Vallet-Regí, M. (2017) Advances in mesoporous silica-based nanocarriers for co-delivery and combination therapy against cancer. Expert Opin. Drug Deliv. 14, 229-243.

(15) Wong, R. C. H., Ng, D. K. P., Fong, W.-P., Lo, P.-C. (2017) Encapsulating $\mathrm{pH}$-Responsive Doxorubicin-Phthalocyanine Conjugates in Mesoporous Silica Nanoparticles for Combined Photodynamic Therapy and Controlled Chemotherapy. Chem. A Eur. J. 23, 16505-16515.

(16) Castillo, R. R., Baeza, A., Vallet-Regí, M. (2017) Recent applications of the combination of mesoporous silica nanoparticles with nucleic acids: development of bioresponsive devices, carriers and sensors. Biomater. Sci. 5, 353-377.

(17) Wang, X.; Li, X.; Yoshiyuki, K.; Watanabe, Y.; Sogo, Y.; Ohno, T.; Tsuji, N. M.; Ito, A. (2016) Comprehensive Mechanism

\section{ACKNOWLEDGMENTS}


Analysis of Mesoporous-Silica-Nanoparticle-Induced Cancer Immunotherapy. Adv. Healthc. Mater. 5, 1169-1176.

(18) Baeza, A., Guisasola, E., Ruiz-Hernández, E., Vallet-Regí, M. (2012) Magnetically Triggered Multidrug Release by Hybrid Mesoporous Silica Nanoparticles. Chem. Mater. 24, 517-524.

(19) Sanchez-Salcedo, S., Vallet-Regí, M., Shahin, S. A., Glackin, C. A., Zink, J. I. (2018) Mesoporous core-shell silica nanoparticles with anti-fouling properties for ovarian cancer therapy. Chem. Eng. J. 340, 114-124.

(20) Liao, L.; Liu, J.; Dreaden, E. C.; Morton, S. W.; Shopsowitz, K. E.; Hammond, P. T.; Johnson, J. A. (2014) A Convergent Synthetic Platform for Single-Nanoparticle Combination Cancer Therapy: Ratiometric Loading and Controlled Release of Cisplatin, Doxorubicin, and Camptothecin. J. Am. Chem. Soc. 136, 5896-5899.

(21) Liu, J.; Luo, Z.; Zhang, J.; Luo, T.; Zhou, J.; Zhao, X.; Cai, K. (2016) Hollow mesoporous silica nanoparticles facilitated drug delivery via cascade $\mathrm{pH}$ stimuli in tumor microenvironment for tumor therapy. Biomaterials 83, 51-65.

(22) Yan, J.; Wang, Y.; Jia, Y.; Liu, S.; Tian, C.; Pan, W.; Liu, X.; Wang, H. (2017) Co-delivery of docetaxel and curcumin prodrug via dual-targeted nanoparticles with synergistic antitumor activity against prostate cancer. Biomed. Pharmacother. 88, 374-383.

(23) Pushpalatha, R., Selvamuthukumar, S., Kilimozhi, D. (2017) Nanocarrier mediated combination drug delivery for chemotherapy - A review. J. Drug Deliv. Sci. Technol. 39, 362-371.

(24) Gottesman, M. M. (2002) Mechanisms of Cancer Drug Resistance. Annu. Rev. Med. 53, 615-627.

(25) Kemp, J. A., Shim, M. S., Heo, C. Y., Kwon, Y. J. (2016) 'Combo' nanomedicine: Co-delivery of multi-modal therapeutics for efficient, targeted, and safe cancer therapy. Adv. Drug Deliv. Rev. 98, 3-18.

(26) Hu, Q., Sun, W., Wang, C., Gu, Z. (2016) Recent advances of cocktail chemotherapy by combination drug delivery systems. Adv. Drug Deliv. Rev. 98, 19-34.

(27) Teo, P. Y., Cheng, W., Hedrick, J. L., Yang, Y. Y. (2016) Codelivery of drugs and plasmid DNA for cancer therapy. Adv. Drug Deliv. Rev. 98, 41-63.

(28) Vallet-Regi, M., Rámila, A., del Real, R. P., Pérez-Pariente, J. (2001) A New Property of MCM-41: Drug Delivery System. Chem. Mater. 13, 308-311.

(29) Baeza, A., Vallet-Regi, M. (2018) Targeted Mesoporous Silica Nanocarriers in Oncology. Curr. Drug Targets 19, 213-224.

(30) Vallet-Regí, M., Balas, F., Arcos, D. (2007) Mesoporous Materials for Drug Delivery. Angew. Chemie Int. Ed. 46, 75487558.

(31) Tang, F., Li, L., Chen, D. (2012) Mesoporous silica nanoparticles: Synthesis, biocompatibility and drug delivery. Adv. Mater. 24, 1504-1534.
(32) Baeza, A., Manzano, M., Colilla, M., Vallet-Regí, M. (2016) Recent advances in mesoporous silica nanoparticles for antitumor therapy: our contribution. Biomater. Sci. 4, 803-813.

(33) Paris, J. L., Colilla, M., Izquierdo-Barba, I., Manzano, M., Vallet-Regí, M. (2017) Tuning mesoporous silica dissolution in physiological environments: a review. J. Mater. Sci. 52, 8761-8771.

(34) Wang, K.; Qi, M.; Guo, C.; Yu, Y.; Wang, B.; Fang, L.; Liu, M.; Wang, Z.; Fan, X.; Chen, D. (2018) Novel Dual Mitochondrial and CD44 Receptor Targeting Nanoparticles for Redox StimuliTriggered Release. Nanoscale Res. Lett. 13, 32.

(35) Li, W.; Guo, Z.; Zheng, K.; Ma, K.; Cui, C.; Wang, L.; Yuan, Y.; Tang, Y. (2017) Dual targeting mesoporous silica nanoparticles for inhibiting tumour cell invasion and metastasis. Int. J. Pharm. 534, 71-80.

(36) López, V.; Villegas, M. R.; Rodríguez, V.; Villaverde, G.; Lozano, D.; Baeza, A.; Vallet-Regí, M. (2017) Janus Mesoporous Silica Nanoparticles for Dual Targeting of Tumor Cells and Mitochondria. ACS Appl. Mater. Interfaces 9, 26697-26706.

(37) Sakhrani, N. M., Padh, H. (2013) Organelle targeting: third level-of drug targeting. Drug Des. Devel. Ther. 7, 585-599.

(38) Field, L. -D., Delehanty, J. B., Chen, Y., Medintz, I. L. (2015) Peptides for specifically targeting nanoparticles to cellular organelles: Quo vadis? Acc. Chem. Res. 48, 1380-1390.

(39) Breunig, M., Bauer, S., Goepferich, A. (2008) Polymers and nanoparticles: Intelligent tools for intracellular targeting? Eur. J. Pharm. Biopharm. 68, 112-128.

(40) Martínez-Carmona, M., Lozano, D., Baeza, A., Colilla, M., Vallet-Regí, M. (2017) A novel visible light responsive nanosystem for cancer treatment. Nanoscale 9, 15967-15973.

(41) Martínez-Carmona, M., Lozano, D., Colilla, M., Vallet-Regí, M. (2018) Lectin-conjugated $\mathrm{pH}$-responsive mesoporous silica nanoparticles for targeted bone cancer treatment. Acta Biomater. 65, 393-404.

(42) Dongbang, S.; Jeon, H. M.; Lee, M. H.; Shin, W. S.; Kwon, J. K.; Kang, C.; Kim, J. S. (2014) Camptothecin delivery into hepatoma cell line by galactose-appended fluorescent drug delivery system. RSC Adv. 4, 18744-18748.

(43) Li, S., Zeng, D. (2007) Chemoenzymatic Enrichment of Phosphotyrosine-Containing Peptides. Angew. Chemie Int. Ed. 46, 4751-4753.

(44) Liu, Y., Pei, Q., Chen, L., Li, Z., Xie, Z. (2016) Reductionresponsive fluorescence off-on BODIPY-camptothecin conjugates for self-reporting drug release. J. Mater. Chem. B 4, 23322337.

(45) Shah, S.; Chandra, A.; Kaur, A.; Sabnis, N.; Lacko, A.; Gryczynski, Z.; Fudala, R.; Gryczynski, I. (2017) Fluorescence properties of doxorubicin in PBS buffer and PVA films. J. Photochem. Photobiol. B Biol. 170, 65-69. 


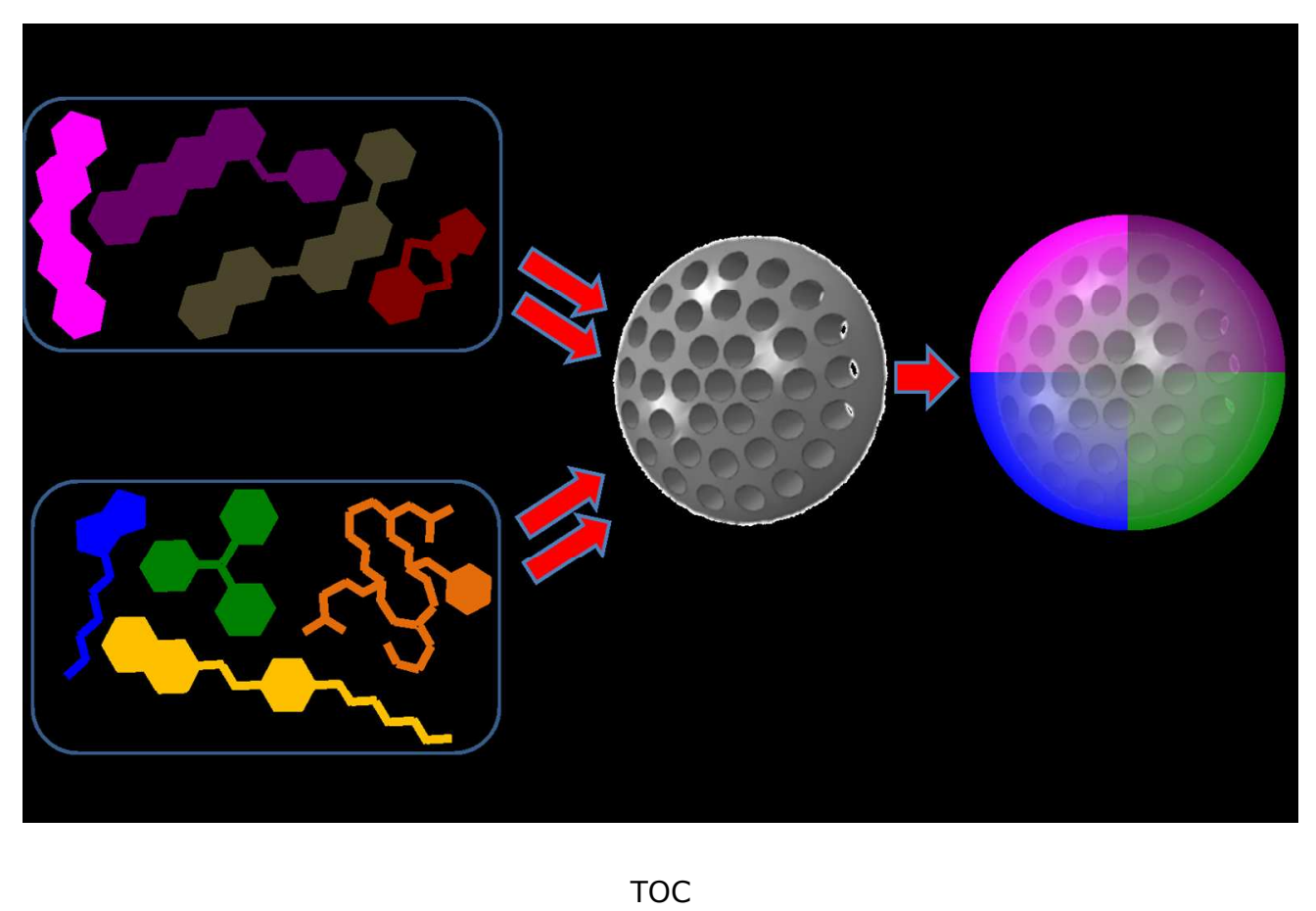

$272 \times 171 \mathrm{~mm}(150 \times 150 \mathrm{DPI})$ 


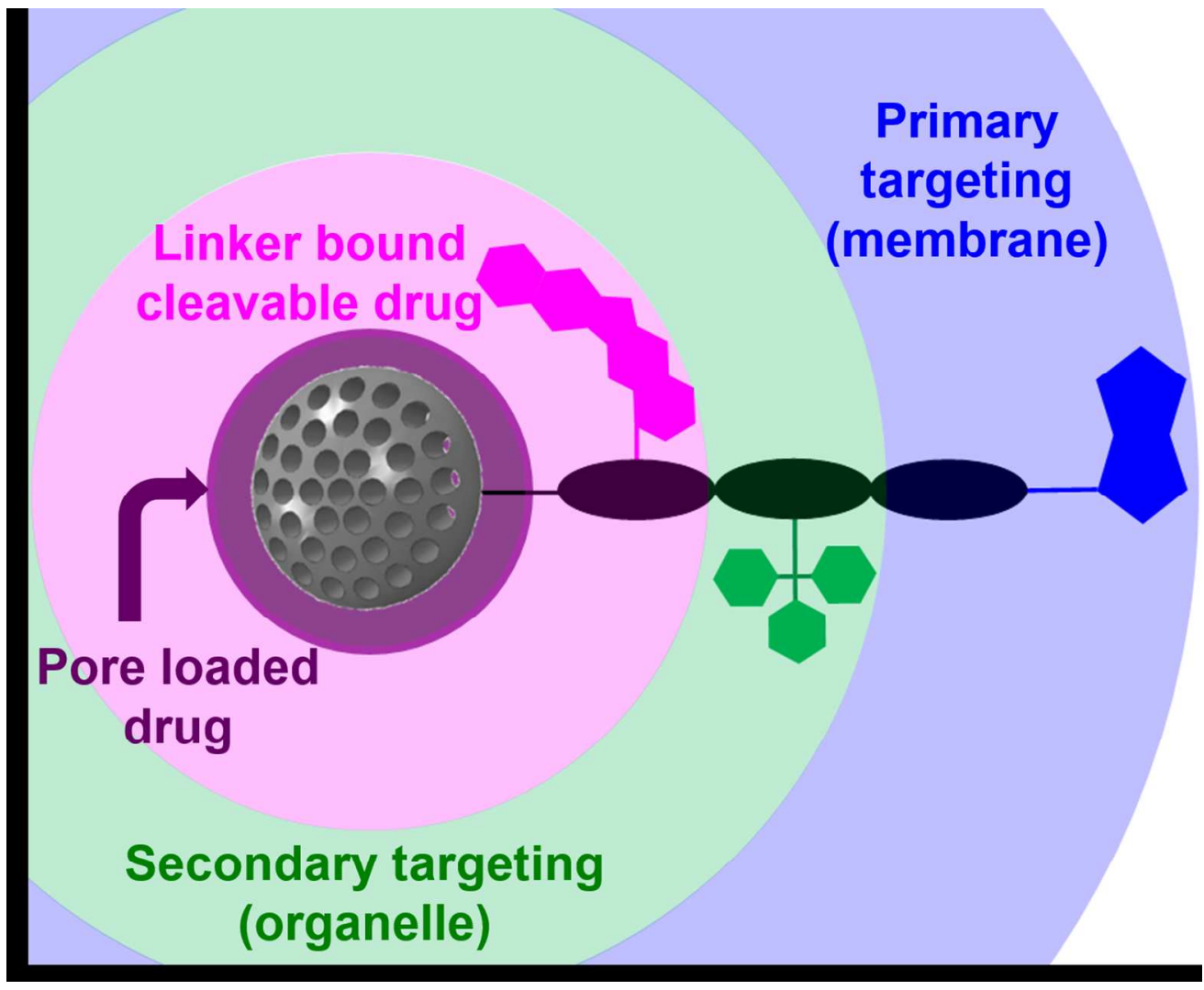

Figure 1. The multilayer strategy employed for the devel-opment of dual targeted two-drug nanocarriers based on mesoporous silica nanoparticles.

$237 \times 193 \mathrm{~mm}(150 \times 150 \mathrm{DPI})$ 


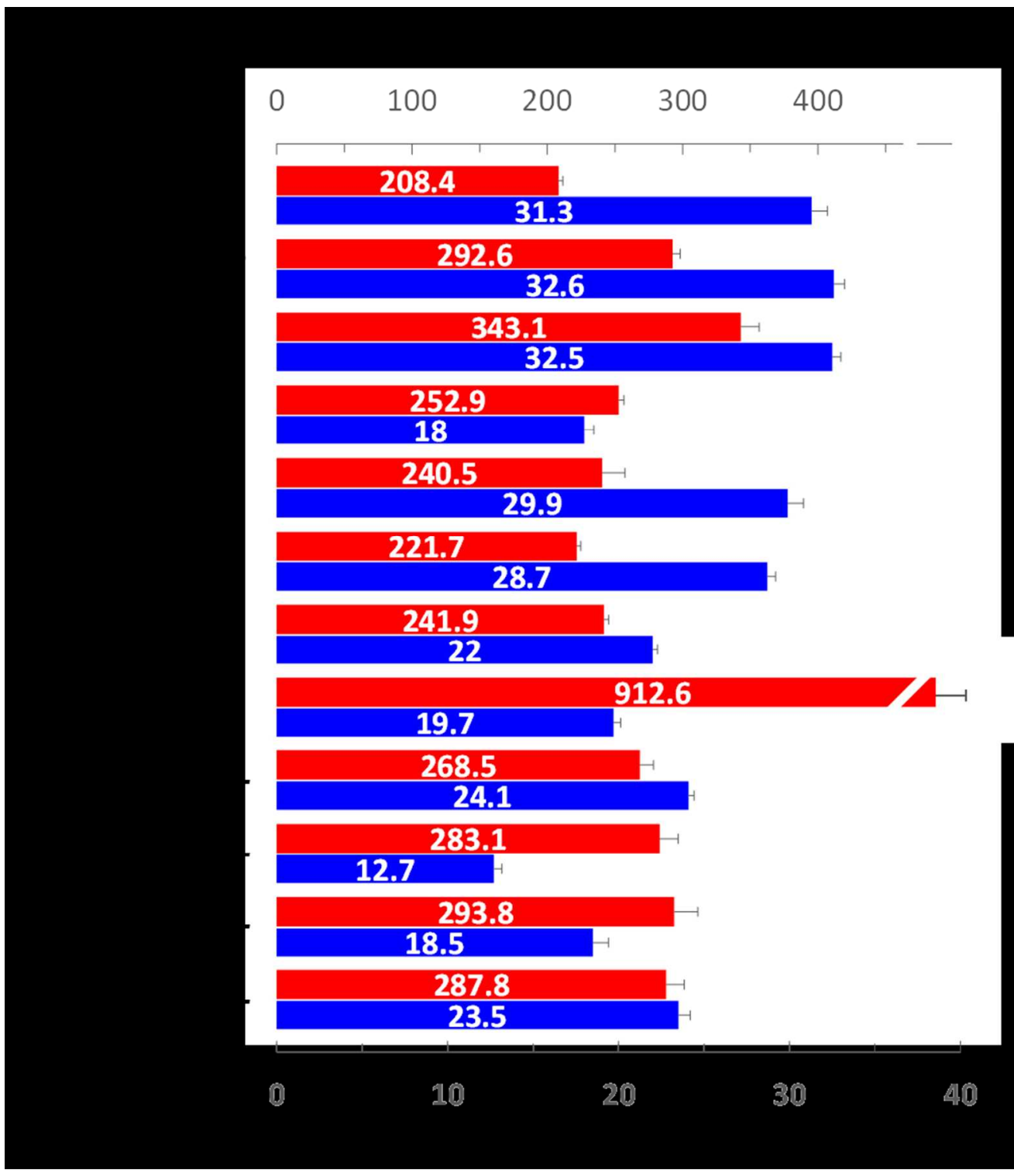

Figure 2. Hydrodynamic diameters (red) and z-potential values (blue) obtained by Dynamic Light Scat-tering (DLS) for all reported hybrids. Data showed correspond to a mean of 5 measures and were record-ed in distilled water. Please check Figures S3 and S4 in the supporting information for raw data of these measurements. Dual refers to TPP-Biotin double targeted ligands with the indicated connectivity. Values for DOXloaded particles could be found on figure S5.

$$
169 \times 195 \mathrm{~mm}(150 \times 150 \mathrm{DPI})
$$




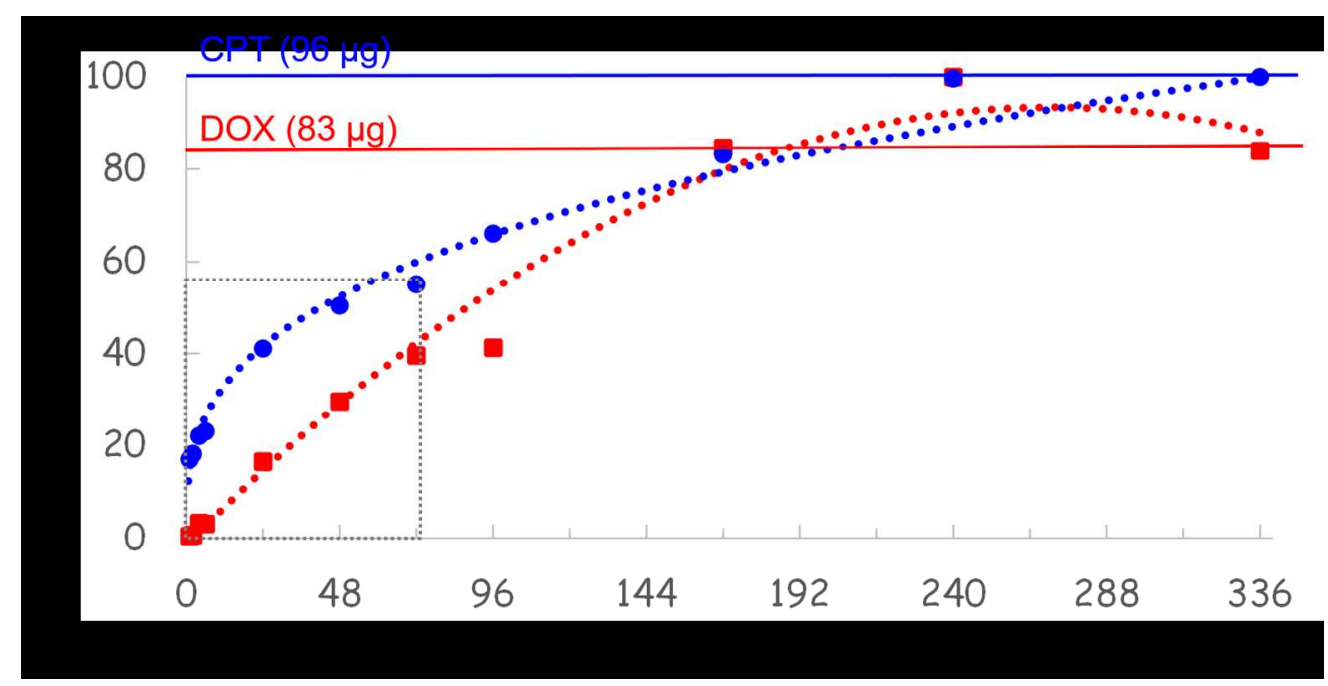

Figure 3. Simultaneous drug release obtained for DOX@F*MSN-Lys(SSLinker-CPT)-Lys(TPP)GABA-Biotin obtained at $37^{\circ} \mathrm{C}$ during 14 days. The $\mu$ g quantities noted on the release graph express the interpolated release values adjusted for the actual amount of hybrid employed. The calculated $96 \mu \mathrm{g}$ estimated for CPT, correspond to a functionalization yield of about $9 \%$ (referenced to the maximum number of available $\mathrm{NH} 2$ groups, supporting information).

$261 \times 133 \mathrm{~mm}(150 \times 150 \mathrm{DPI})$ 


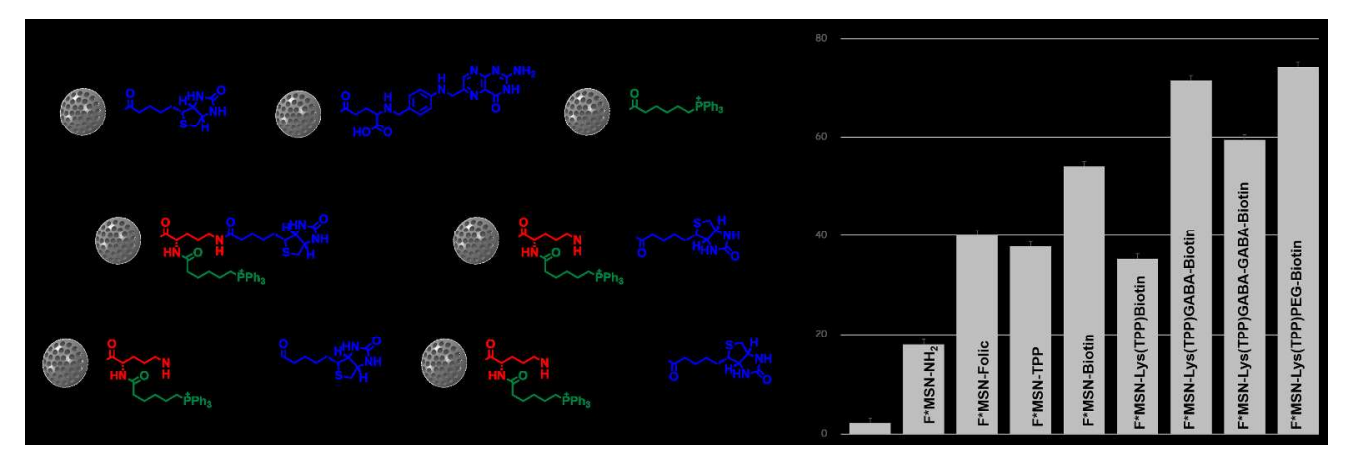

Figure 4. Prepared single and double targeted fluorescein labeled organosilica nanohybrids. Cell uptake measured by flow cytometry at $2 \mathrm{~h}$ of internalization in HOS cells. Data are mean \pm SEM of 3 independent experiments performed in triplicate $* \mathrm{p}<0.05$ vs HOS control; **p $<0.05$ vs $\mathrm{F}^{*} \mathrm{MSN}-\mathrm{NH} 2 ; * * * \mathrm{p}<0.01$ vs $\mathrm{F}^{*} \mathrm{MSN}-\mathrm{NH} 2 ; \# \mathrm{p}<0.01$ vs all groups.

$573 \times 187 \mathrm{~mm}(150 \times 150$ DPI) 


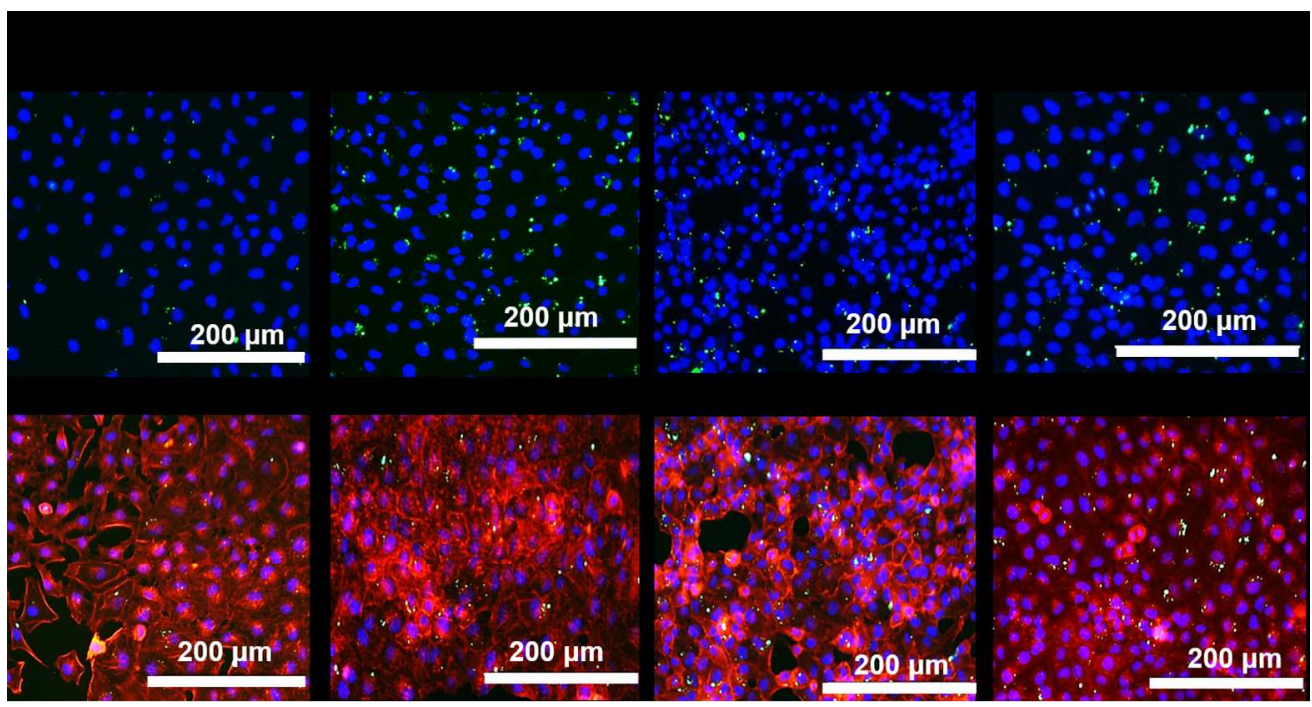

Figure 5. Representative fluorescence microscopy images of HOS cells incubated with fluorescein labeled nanoparticles at $2 \mathrm{~h}$ of internalization. Blue fluorescence (nuclei), red (actin filaments) and green fluorescence (fluorescein labeled nanoparticles).

$265 \times 140 \mathrm{~mm}(150 \times 150 \mathrm{DPI})$ 
Figure 6. Cytotoxicity assay measured by Alamar Blue in HOS cells with the different nanoparticles loaded with DOX, CPT or both at $24 \mathrm{~h}(\mathrm{~A}) 48 \mathrm{~h}(\mathrm{~B})$ and $72 \mathrm{~h}(\mathrm{C})$ of cell culture. Data are mean \pm SEM of 3 independent experiments per-formed in triplicate. ${ }^{*} p<0.05$ vs corresponding non-targeted, Biotin or dual nanoparticles. NONE/DOX, particles refer to DOX loaded F*MSN-NH2 (DOX@F*MSN-NH2), NONE/CPT refers to $\mathrm{F}^{*} \mathrm{MSN}$-Lys(SSLinker-CPT)NH2 and NONE/CPT+DOX to DOX@F*MSN-Lys(SSLinker-CPT)NH2. Notation employed for other hybrids is equivalent, with the proviso that Dual refers to Lys(TPP)GABA-Biotin targeting ligand.

$278 \times 187 \mathrm{~mm}(150 \times 150 \mathrm{DPI})$ 
Figure 7. Cytotoxicity assay measured by Alamar Blue in HOS cells for differently targeted MOSHs (4-NO2Bz, Biotin and Lys(TPP)GABA-Biotin) with DOX, CPT or both at $48 \mathrm{~h}$ of cell culture. Data are mean \pm SEM of 3 independent experiments performed in triplicate. ${ }^{*} \mathrm{p}<0.05$ vs corresponding 4-NO2Bz, (4-Nitrobenzoyl) Biotin or dual targeted hybrids; \#p < 0.001 vs corresponding 4-NO2Bz, Biotin or dual targeted hybrids. Dual refers to Lys(TPP)GABA-Biotin targeting ligand.

$261 \times 110 \mathrm{~mm}(150 \times 150 \mathrm{DPI})$ 